\title{
Estudio de las propiedades al creep en aceros termorresistentes.
}

\section{Study of creep properties of heat resistant steels}

Presentación: 06/10/2020

\section{Doctorando:}

\section{Gabriel González}

Grupo de Estudio sobre Materiales, Facultad Regional Bahía Blanca, Universidad Tecnológica Nacional - Argentina

ggonzal@frbb.utn.edu.ar

\section{Director/es:}

\section{Lilian Moro}

\section{Co-director/es:}

\section{Lucio Iurman}

\begin{abstract}
Resumen
Cuando los componentes de una planta industrial se encuentran sometidos a condiciones severas de temperatura y esfuerzos mecánicos, sufren una deformación plástica que continua a lo largo del tiempo. Este proceso, denominado termofluencia (creep), se presenta en industrias petroquímicas, plantas generadoras de energía y refinerías de petróleo, donde sus componentes son utilizados durante largos períodos de tiempo y expuestos a condiciones severas de servicio.

El objetivo de esta tesis es estudiar el comportamiento al creep de aceros ferríticos (1Cr 0.5Mo, $2.25 \mathrm{Cr} 1 \mathrm{Mo}$ y $5 \mathrm{Cr} 0.5 \mathrm{Mo})$, y aleaciones base níquel ((Incoloy $800(30 \mathrm{Ni}-20 \mathrm{Cr}$ ), Inconel $601(60 \mathrm{Ni}-25 \mathrm{Cr}$ ) y aceros de la serie $\mathrm{HP}(25 \mathrm{Cr}-35 \mathrm{Ni})$ que poseen, alta resistencia termomecánica asociada a la microestructura interna de la matriz. Se analiza la variación de los parámetros característicos al creep, tales como la energía de activación y el coeficiente de tensión, relacionados con la velocidad de deformación, la tensión aplicada y la temperatura; simultáneamente se caracteriza la evolución microestructural, en las condiciones de temperatura y tiempo similares a los ensayos.

En forma experimental, mediante ensayos de creep, se reproducirán condiciones de ensayo en el laboratorio, de características similares a las de operación en servicio. Las curvas experimentales obtenidas de esta forma, nos van a permitir conocer la variación de las propiedades físicas y mecánicas; estudiar los mecanismos responsables del fenómeno y describir parámetros que estén vinculados con la resistencia mecánica del material.
\end{abstract}

Palabras clave: aceros ferriticos, aleaciones base níquel, alta temperatura, termofluencia.

\footnotetext{
Abstract

In industrial plant the components are subjected to severe conditions of temperature and mechanical stress, the plastic deformation that continues over time. This process, called creep, occurs in petrochemical industries, power plants, and oil refineries, where its components are used for long periods of time and exposed to severe service conditions.

The objective of this thesis is to study the creep behavior of ferritic steels $(1 \mathrm{Cr} 0.5 \mathrm{Mo}, 2.25 \mathrm{Cr} 1 \mathrm{Mo}$ and $5 \mathrm{Cr} 0.5$ Mo), and nickel-based alloys ((Incoloy 800 (30 Ni - $20 \mathrm{Cr}$ ), Inconel 601 (60 Ni - 25 Cr) and steels of the HP series (25 $\mathrm{Cr}-35 \mathrm{Ni}$ ) that have high thermomechanical resistance associated with the internal microstructure of the matrix. The variation of the characteristic parameters of creep, such as activation energy and coefficient of stress, related to the deformation rate, the applied stress and the temperature; simultaneously the microstructural evolution is characterized, in the conditions of temperature and time similar to the tests.

Experimentally, through creep tests, test conditions will be reproduced in the laboratory, with characteristics similar to those of in-service operation. The experimental curves obtained in this way will allow us to know the variation of the physical and mechanical properties; study the mechanisms responsible for the phenomenon and describe parameters that are related to the mechanical resistance of the material.
} 
Keywords: ferritic steels, nickel base alloys, high temperature, creep.

\section{Introducción}

Cuando los componentes de una planta industrial se encuentran sometidos a condiciones severas de temperatura y esfuerzos mecánicos, sufren una deformación plástica que continúa a lo largo del tiempo. Este proceso, denominado termofluencia o fluencia lenta (creep), se evidencia a partir de transformaciones en la microestructura del material y la pérdida de la resistencia mecánica (Viswanathan, R, 1989).

El estudio de este fenómeno adquiere aún mayor importancia, cuando los materiales afectados son aceros destinados a industrias petroquímicas, plantas generadoras de energía y refinerías de petróleo, ya que sus componentes son utilizados durante largos períodos de tiempo y expuestos a condiciones severas de servicio. La importancia que tiene la comprensión del comportamiento de los materiales para el diseño industrial, se debe principalmente al alto costo que representa para las industrias la rotura de sus equipos, problema que puede ser solucionado cuando se realiza una correcta selección del material de sus componentes, tratamientos térmicos y/o termoquímicos, o estimando el tiempo de vida residual de los mismos.

El objetivo de esta tesis es estudiar el comportamiento al creep de aceros ferríticos, y aleaciones base níquel que posee alta resistencia termomecánica asociada a la microestructura interna de la matriz. Se analiza la variación de los parámetros característicos al creep, tales como la energía de activación y el coeficiente de tensión, relacionados con la velocidad de deformación, la tensión aplicada y la temperatura; simultáneamente se caracteriza la evolución microestructural, en las condiciones de temperatura y tiempo similares a los ensayos.

Se estudia el comportamiento al creep de aceros ferríticos, con distinto contenido de $\mathrm{Cr}(1 \mathrm{Cr} 0.5 \mathrm{Mo}, 2.25 \mathrm{Cr} 1 \mathrm{Mo}$ y $5 \mathrm{Cr} 0.5 \mathrm{Mo}$ ), y aleaciones base $\mathrm{Ni}$ (Incoloy $800(30 \mathrm{Ni}-20 \mathrm{Cr}$ ), Inconel $601(60 \mathrm{Ni}-25 \mathrm{Cr}$ ) y aceros de la serie HP $(25 \mathrm{Cr}-35 \mathrm{Ni})$ y se observa la influencia sobre la resistencia al creep de la composición química y de la evolución microestructural; ya que una mayor durabilidad de los materiales representa una gran ventaja económica.

Los acero ferrifico poseen una estructura con finos carburos aleados que impiden el movimiento de las dislocaciones; sin embargo es inevitable, que durante largos períodos de servicio o condiciones muy críticas, se produzcan transformaciones que provocan la pérdida de resistencia del material. Entre las modificaciones de la estructura se puede mencionar: precipitación y transformación de carburos, descomposición de áreas de perlita/bainita, cambios en la morfología de los carburos y variación en la composición química de la matriz. El predominio de alguna transformación determinada, dependerá de la microestructura que presenta el material en estado original. Badeshia y Thompson estudiaron la morfología de aceros ferríticos, luego de haber sido sometidos a distintos tratamientos térmicos y encontraron que los carburos precipitados, cuando la muestra inicial tenía estructura bainítica, diferían de los obtenidos a partir de un material con una estructura inicial compuesta por bainita y ferrita alotromórfica (Thomson, R.C, 1992).

Mientras que las aleaciones de niquel presentan una microestructura constituida por una matriz formada por una solución sólida fcc (fase $\gamma$ ) y un disperso de precipitados intermetálicos (fase $\gamma^{\prime}$ ) que son los responsables de su gran resistencia. En particular el acero HP modificado, se utiliza ampliamente en las industrias petroquímicas de nuestra región, para construir los hornos de pirolisis porque presentan resistencia a la termofluencia gracias a la estabilidad de las fases que lo componen (Alessio D, 2012).

\section{Desarrollo y método experimental}

\section{Materiales estudiados}

Se estudian dos tipos de materiales, aceros ferríticos con bajo contenido de $\mathrm{Cr}$ y aleaciones base Ni que poseen un alto contenido de Cr. Los aceros ferríticos son usados en las plantas generadoras de energía en carcasas de turbinas de vapor y colectores de alta presión. Son aceros de baja aleación, resistentes al creep, que por lo general son puestos en servicio luego de un tratamiento térmico de normalizado. La microestructura inicial depende del tratamiento térmico recibido durante su proceso de fabricación y presenta distintos precipitados que le confieren mayor durabilidad. Mientras que las aleaciones base níquel presentan gran resistencia al calor y son capaces de operar a temperaturas que pueden llegar hasta un $85 \%$ de su punto de fusión. Por este motivo se destinan a aplicaciones en las que se requiere que el material presente una excelente combinación de propiedades mecánicas, tales como: elevadas tensiones de fluencia y de rotura, buena resistencia a la fatiga y al creep, resistente a diferentes tipos de corrosión; y que sea apto para trabajar a elevadas temperaturas durante largos períodos de tiempo. 
En primer lugar se analizó el acero ferrifico $1 \mathrm{Cr}-0,5$ Mo, se determinó la composición química del material mediante un espectrómetro de emisión por plasma, marca Spectromax modelo X. En la Tabla 1, se presentan los elementos componentes y los valores medidos de las concentraciones (\% en peso) del material.

\begin{tabular}{|c|c|c|c|c|c|c|c|c|c|c|}
\hline Material & $\mathbf{C}$ & $\mathbf{C r}$ & $\mathbf{M o}$ & $\mathbf{V}$ & $\mathbf{N i}$ & $\mathbf{M n}$ & $\mathbf{S i}$ & $\mathbf{C u}$ & $\mathbf{O t r o s}$ & $\mathbf{F e}$ \\
\hline $\begin{array}{c}\text { ASTM A297 } \\
\text { Grado HP }\end{array}$ & 0.06 & 1.01 & 0.46 & -- & 0.10 & 0.43 & 0.85 & 0.11 & $\begin{array}{c}\mathrm{S}, \mathrm{Sn}< \\
0.011\end{array}$ & Balance \\
\hline
\end{tabular}

Tabla 1: Composición química del acero $1 \mathrm{Cr}-0.5$ Mo.

Se estudió la microestructura del material en su estado original por medio de la observación con microscopía óptica, previamente las muestras fueron desbastados con papeles abrasivos de diferentes granulometrías y atacados químicamente por inmersión, en Nital al 2\%. En la Figura 1 se muestra la microestructura del acero en su forma de partida, allí se observa una estructura con una matriz ferrítica de grano fino y con áreas oscuras que corresponden a perlita y/o bainita.

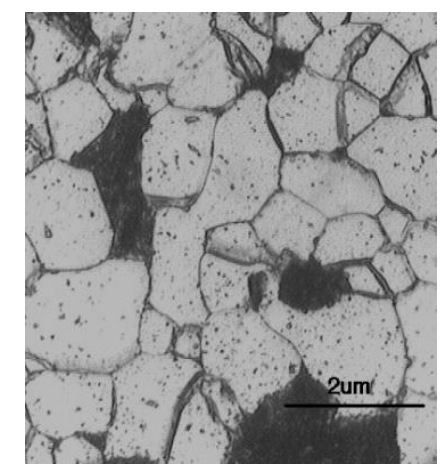

Figura 1: Micrografías de la estructura del acero 1Cr-0.5Mo en estado de recepción (600X)

En forma similar se estudió dentro de las aleaciones de níquel al acero HP modificado, en la Tabla 2 se indica la composición química donde se presentan los elementos componentes y las concentraciones (\% en peso). En la figura 2, se presenta la microestructura correspondiente al acero HP - modificado con Nb, en su condición as-cast, para ello, las probetas fueron desbastadas con papeles abrasivos de diferentes granulometrías y atacadas químicamente por inmersión, con Gliceregia $\left(60 \% \mathrm{HCl}, 20 \% \mathrm{HNO}_{3}, 40 \%\right.$ glicerol), durante 25 segundos a temperatura ambiente. El estudio de la microestructura permite interpretar las transformaciones cinéticas que ocurren en las fases precipitadas, la nucleación y evolución de microcavidades responsables del daño.

Se puede apreciarse, que la microestructura es de tipo dendrítica compuesta por una matriz austenítica $\gamma$ y carburos primarios del tipo eutéctico, ricos en $\mathrm{Cr}$ (del tipo $\mathrm{M}_{7} \mathrm{C}_{3}$ y/o $\mathrm{M}_{23} \mathrm{C}_{6}$ ) y carburos de niobio del tipo $\mathrm{MC}$, ubicados entre las dendritas junto a una leve dispersión intragranular.

\begin{tabular}{|c|c|c|c|c|c|c|c|c|c|c|c|c|}
\hline Material & Ni & Cr & Fe & C & Mn & Si & Ti & Nb & Mo & W & P & S \\
\hline $\begin{array}{c}\text { ASTM A297 } \\
\text { Grado HP }\end{array}$ & 34.2 & 27.1 & 34.2 & 0.045 & 1.75 & 0.04 & 0.59 & 0.06 & 0.05 & 0.14 & 0.27 & 0.009 \\
\hline
\end{tabular}

Tabla 2: Composición química del acero HP - modificado.

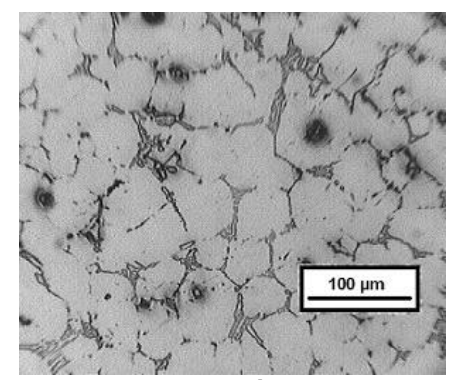

Figura 2: Microestructura característica del acero HP- modificado.

\section{Método de ensayo de creep}

El equipo para realizar los ensayos de creep fue diseñado y construido íntegramente en la FRBB, consta de un sistema de tres máquinas, de igual diseño, que someten al material bajo ensayo a tensión y temperatura constantes, 
manteniendo la independencia de funcionamiento de cada una de ellas con la particularidad de estar controlado por un único sistema de adquisición y software. El equipo está formado por una barra principal, apoyada en un extremo a un amortiguador regulable, y en el otro extremo posee una leva circular de radio constante. De la barra principal cuelga un peso variable, de trazabilidad controlada, que se desplaza a lo largo de la misma, permitiendo modificar la distancia al centro de rotación y así poder lograr la tensión que se desea aplicar. El peso está ubicado en un carro con rodamientos que permiten el desplazamiento del mismo a lo largo de la barra. El movimiento se realiza mediante un tornillo patrón impulsado por un moto-reductor de corriente continua. El equipo tiene anexado un horno eléctrico, donde el control de la temperatura se efectúa por medio de una termocupla de cromel alumel, cuyo extremo caliente se ubica sobre la superficie de la zona central calibrada de la probeta. La deformación de la zona calibrada de la probeta se mide por medio de dos transformadores lineales de diferencial variable (LVDT) Marca Solartron, que produce una señal adquirida por un sistema analógico- digital (Molina R, 2018).

Estos ensayos se realizaron respetando las condiciones de la norma (ASTM E139, 2018).

A partir de los datos registrados en los ensayos de creep realizados a una misma tensión, se calculó la energía de activación graficando la velocidad de deformación en función de la inversa de la temperatura. De igual forma se obtuvo el valor $\mathrm{n}$ de tensión graficando la velocidad de deformación $\dot{\varepsilon}$, en función de la tensión $\sigma$, a una misma temperatura de ensayo.

\section{Resultados obtenidos}

Se realizaron ensayos de de creep sobre el acero $1 \mathrm{Cr} 0,5$ Mo a una tensión de 168 MPA y a temperaturas de 843, 873 y 923K, luego con la finalidad de mejorar la resistencia al creep en el acero se realizaron tratamientos térmicos que consistió en una austenización a $1223 \mathrm{~K}$ seguida de un enfriamiento en aire y luego un revenido a 1023K durante 3 horas y enfriamiento nuevamente al aire en probetas de material en estado de recepción. En la Figura 4 se presenta las gráficas en función del tiempo a para el material sin tratamiento térmico y para el material tratado térmicamente.

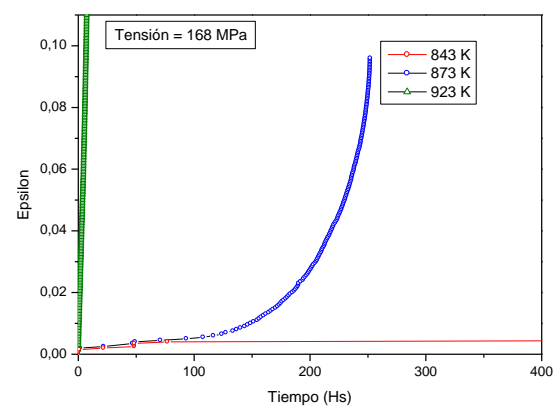

a)

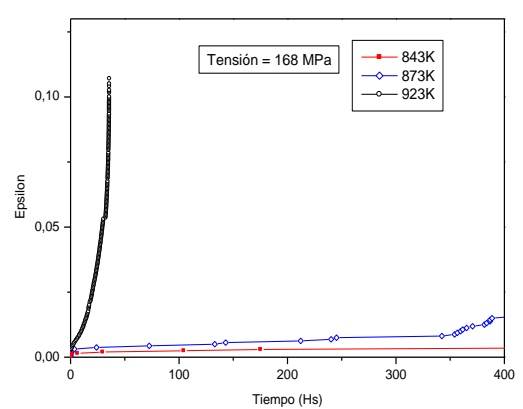

b)

Figura 4: Gráfica de la deformación especifica por creep en función del tiempo. a) material en estado de recepción b) material tratado térmicamente

A partir de las gráficas trazadas se calcularon las velocidades de deformación de la zona del creep secundario (estado estacionario) donde la pendiente de la curva se mantiene prácticamente constante. Con estos valores para la tensión equivalente de 168 MPa y temperaturas de 873, 893 y 1023K se calculó el valor de la energía de activación; y con un procedimiento similar, utilizando los datos obtenidos de los ensayos realizados a la temperatura de $873 \mathrm{~K} \mathrm{y}$ tensiones de 82, 168 y 205 MPa se obtiene un valor del exponente de tensión Tabla 3.

\begin{tabular}{|c|c|c|}
\hline $\begin{array}{c}\text { Acero 1Cr- 0,5 } \\
\text { Mo }\end{array}$ & $\begin{array}{c}\text { Sin tratamiento } \\
\text { térmico }\end{array}$ & $\begin{array}{c}\text { Con tratamiento } \\
\text { térmico }\end{array}$ \\
\hline $\begin{array}{c}\text { Energía de } \\
\text { activación }\end{array}$ & 764 & 418 \\
\hline $\begin{array}{c}\text { Coeficiente de } \\
\text { tensión }\end{array}$ & 2.13 & 7.15 \\
\hline
\end{tabular}

Tabla 3: Valores de la energía de activación y de los coeficientes de tensión.

En la figura 5 se muestra el estudio de la microestructura realizado con un microscopio electrónico de barrido JEOL JSM 35, en el mismo se indica la evolución de la estructura del material ensayado a una temperatura $\mathrm{T}=873 \mathrm{~K}$ una tensión de $168 \mathrm{MPa}$. En la figura 5 (a), se observa el material al finalizar la etapa de creep primario, donde se 
evidencian los precipitados finos de los carburos en el interior y en los bordes de grano; solo aparecen pequeños huecos en los bordes de grano que son perpendiculares al eje de la tensión. En la figura 5 (b), se observa el material en la etapa secundaria donde se evidencia la formación de cadenas de cavidades o huecos lo que indica que se inicia el tipo de fractura transgranular. Los carburos precipitados en el interior y en los bordes de los granos, se agrupan, disminuye su dispersión y aumenta su tamaño. Mientras que en la figura 5 (c) se expone el material previo a iniciarse la etapa terciaria, donde se observa la fractura transgranular, en esta etapa los carburos precipitados en borde de granos evidencian un tamaño importante.

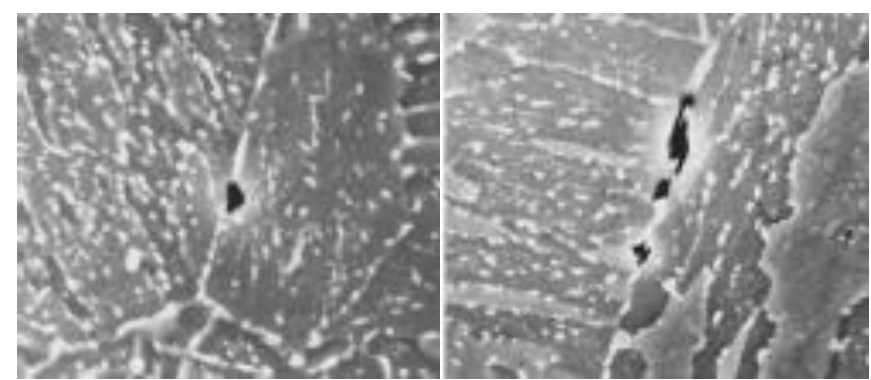

(a)

(b)

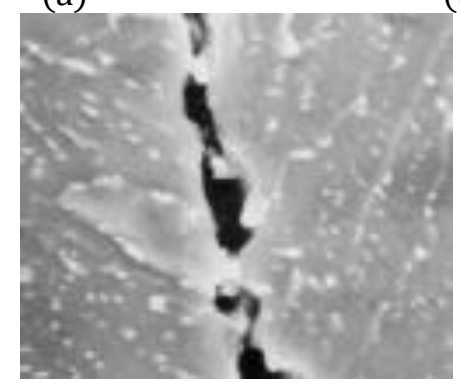

(c)

Figura 5: Evolución de la microestructura de una muestra sometida a creep a T= $873 \mathrm{~K}$ y tensión $168 \mathrm{MPa}$ (5000x)

A fin de evaluar la influencia del $\mathrm{Cr}$ en la resistencia al creep del material se realizaron ensayos de creep a una tensión de $168 \mathrm{MPa}$ y a una temperatura de $873 \mathrm{~K}$ en aceros con un contenido de $\mathrm{Cr}$ de 2,25 y 5, se puede observar como a medida que aumentaba el porcentaje de $\mathrm{Cr}$ se incrementaba la resistencia al creep. (Figura 6).

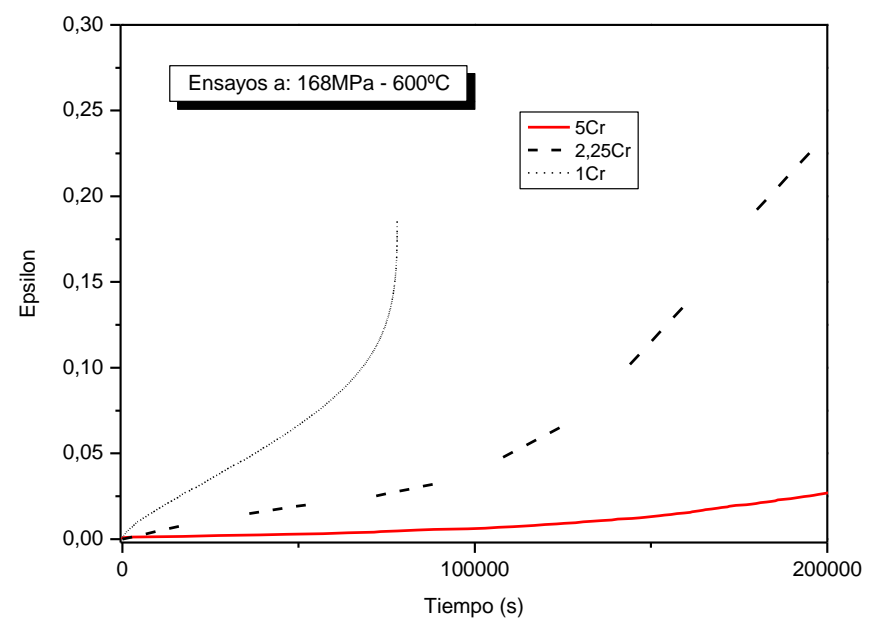

Figura 6: Grafica de la deformación especifica de creep en función del tiempo de aceros con distintos contenidos de Cr.

Posteriormente se estudió las aleaciones base níquel, en particular el material ASTM A297 Gr HP, para poder relacionar el comportamiento del material a distintas tensiones y temperaturas se realizaron ensayos de creep por tracción hasta la rotura, trabajando en un rango de temperaturas entre 1073 y $1273 \mathrm{~K} \mathrm{y} \mathrm{a} \mathrm{tensiones} \mathrm{efectivas} \mathrm{entre}$ 50 y $90 \mathrm{MPa}$. En la Tabla 4 se presentan los valores de que se obtuvieron de los ensayos a una temperatura de 1173 K y tensiones de 50 a 80p MPa. En la Tabla 5 se observan los valores de los ensayos realizados a una tensión de 50 MPa y a temperaturas de 1173 a $1253 \mathrm{~K}$. 


\begin{tabular}{|c|c|c|c|c|}
\hline Tensión (MPa) & $\mathbf{5 0}$ & $\mathbf{6 0}$ & $\mathbf{7 0}$ & $\mathbf{8 0}$ \\
\hline $\begin{array}{c}\text { Duración del ensayo en } \\
(\mathrm{hs})\end{array}$ & 855.79 & 474.59 & 158.53 & 113.16 \\
\hline $\begin{array}{c}\text { Velocidad min de creep } \\
(\% \mathrm{~h})\end{array}$ & $1.48 \times 10^{-2}$ & $1.85 \times 10^{-2}$ & $6.45 .10^{-2}$ & $8.88 \times 10^{-3}$ \\
\hline \begin{tabular}{c} 
Deformación máxima (\%) \\
\hline
\end{tabular} & 3.5 & 20.2 & 23.2 & 13.8 \\
\hline
\end{tabular}

Tabla 4: Valores comparativos de ensayos realizados a una temperatura de $1173 \mathrm{~K}$.

\begin{tabular}{|c|c|c|c|c|}
\hline $\begin{array}{c}\text { Temperatura de } \\
\text { ensayo (K) }\end{array}$ & $\mathbf{1 1 7 3}$ & $\mathbf{1 1 9 3}$ & $\mathbf{1 2 2 3}$ & $\mathbf{1 2 5 3}$ \\
\hline $\begin{array}{c}\text { Duración del ensayo en } \\
\text { (hs) }\end{array}$ & 855.79 & 474.59 & 158.53 & 113.16 \\
\hline $\begin{array}{c}\text { Velocidad min de creep } \\
\text { (\% h) }\end{array}$ & $1.85 \times 10^{-2}$ & $2 \times 10^{-2}$ & $3.05 \times 10^{-3}$ & 0.5369 \\
\hline Deformación máxima (\%) & 20.2 & 10.7 & 17.8 & 26.5 \\
\hline
\end{tabular}

Tabla 5: Valores comparativos de ensayos realizados a una tensión de $60 \mathrm{MPa}$.

A partir de los ensayos y relacionando los valores de la velocidad de deformación por termofluencia para diferentes tensiones mecánicas aplicadas, a una misma temperatura de ensayo, $\mathrm{T}=1173 \mathrm{~K}$, se pueden apreciar, dos regiones bien diferenciadas en la relación entre $\mathrm{d} \varepsilon / \mathrm{dt}$ y $\sigma$. A bajas tensiones, el exponente $\mathrm{n}$ es igual a 6 , mientras que a tensiones más altas, el exponente $\mathrm{n}$ da un valor valor igual a 19.

También, se puede calcular la energía de activación, obteniendo dos valores para la energía de activación; para las temperaturas de ensayo por debajo de los $1193 \mathrm{~K}$, se obtiene un valor de la energía de activación de 331.43 $\mathrm{KJ} / \mathrm{mol}$, mientras que, para valores superiores a esta temperatura de quiebre, la energía de activación se ve incrementada a un valor de $450.47 \mathrm{KJ} / \mathrm{mol}$.

\section{Referencias}

Viswanathan R. (1989). Damage Mechanisms and Life Assessment of High Temperature Components. ASM International Metals, Park Ohio, USA

Thomson, R.C. y Badeshia, H.K.D.H.; (1992). Carbide Precipitation in 12Cr -1MoV Power Plant Steel. Metallurgical Transactions A, 23, 1171-1179. https://doi.org/10.1179/026708310X12635619988302.

Alessio D., Gonzalez G., Fernandez Pirrone V., Iurman L.,Moro L.; (2012). Variation of Creep Properties in HP Steel by Influence of Temperature. Procedia Materials Science, 1, 104-109.

https://doi.org/10.1016/j.mspro.2012.06.014

Molina R., González G., Pender G., Moro, L., (2018), Checking of the uniformity of results of simultaneous creep testing equipment. Revista Materia, 26 N.02, ISSN 1517-7076 artículo e-12022, 2018 .

https://doi.org/10.1590/S1517-707620180002.0358 .

ASTM E139 -11. (2018). Standard Test Methods for Conducting Creep, Creep-Rupture, and Stress-Rupture Tests of Metallic Materials.

https://www.astm.org/Standards/E139.htm. 